\title{
Complex Probiotics Supplementation Improves Overtraining-Induced Muscle Inflammatory Response and Antioxidant Status Via LPS-TLR4/NF- kb Signalling Pathway in Rats
}

\author{
Yali Xia \\ Shanghai University of Sport \\ Jing Shao \\ National Institute of Sports Medicine \\ Hui Wang \\ Shanghai University of Sport \\ Qiushi Tan \\ National Institute of Sports Medicine \\ Qi Han \\ National Institute of Sports Medicine
}

Muqing YI ( $\nabla$ muqingyi@163.com )

National Insitute of Sports Medicine https://orcid.org/0000-0002-4877-0819

Research article

Keywords: Excessive exercise, Probiotics, Inflammatory response, Oxidative stress, TLRs/NF-KB signalling pathway.

Posted Date: May 14th, 2021

DOl: https://doi.org/10.21203/rs.3.rs-513418/v1

License: (c) (i) This work is licensed under a Creative Commons Attribution 4.0 International License. Read Full License 


\section{Abstract}

Background: Prolonged endurance and intensive exercise has shown detrimental effects on muscle health as well as causes intestinal dysbiosis. Probiotics are known as live microorganisms effective in improving gut microbiota and immunity to warrant health and well-being, which exert global and local biological functions on gut and muscle comprising of anti-oxidative stress, anti-inflammatory and antiapoptosis, etc,. This research was conducted to investigate whether complex probiotics supplementation could alleviate excessive exercise-induced muscle damage and underlying signaling mechanism in rats.

Methods: Thirty-two male SD rats were randomly allocated to four groups: control (SC), exercise (EC), probiotics (SP) and exercise with probiotics (EP) ( $n=8$ each). In the overtraining model, rats were arranged to an incremental load training on the treadmill 6 days/week for 8 weeks and gavaged with complex-probiotic-preparation $\left(4 \times 10^{9} \mathrm{CFU} / \mathrm{g}\right.$ ) containing maltodextrin or maltodextrin only once a day. Serum and soleus were collected at the end of experiment. Serum creatine kinase (CK) and lactate dehydrogenase (LDH) activities, the levels of lipopolysaccharide (LPS), interleukin-6 (IL-6), interleukin-1 $\beta$ (IL-1 $\beta$ ), tumor necrosis factor-a (TNF-a), malondialdehyde (MDA), and muscle total antioxidant capacity (T-AOC), glutathione peroxidase (GPx), catalase (CAT) in soleus were measured. Furthermore, soleus protein expression of toll-like receptor 4 (TLR4), myeloid differentiation factor 88 (MyD88), p-p65/p65, Bax, Bcl-2, caspase- 9 and caspase- 3 were determined by western blotting assay.

Results: The findings indicated that excessive exercise induced a significant increased CK and LDH activities, LPS levels, relative protein expression of TLR4, MyD88, p-p65/p65, inflammatory cytokines and lipid peroxidation $(p<0.05)$. Simultaneously, the antioxidant elements including T-AOC, GPx, CAT were markedly decreased in the muscle of EC group $(p<0.05)$. In addition, up-regulated the protein expression of Bax/Bcl-2, cleaved caspase-9/procaspase-9 and cleaved caspase-3/procaspase-3 in muscle $(p<0.05)$. Administration of complex probiotics attenuated overtraining-induced inflammatory response, oxidative injury and TLR4/nuclear factor-kappa B (NF-kB) signaling factors expression as a result of reversing the most of above altered biochemical parameters $(p<0.05)$, and there was only tendency downregulation in the expression of apoptosis-regulated proteins $(p>0.05)$.

Conclusions: Administration of complex probiotics could improve capability of the anti-oxidant, antiinflammatory status induced by excessive exercise in rat skeletal muscle possibly via protecting the gut mucosal barrier, inhibiting the outflow of endotoxin, attenuating the action of TLR4-mediated NF-kB pathway.

\section{Introduction}

Excessive exercise (prolonged or intense or exhaustive exercise) may cause overtraining syndrome (OTS) characterized by exercise fatigue, gastrointestinal dysfunction[1], skeletal muscle injury [2], aggravating inflammatory response[3], weakened immunity[4] and damaged exercise performance[5] in athletes, especially in endurance (e.g., marathons, triathlons, etc.) athletes, in which main symptoms include 
emotional instability, skeletal muscle soreness and swelling, abdominal distension and diarrhea, upper respiratory tract infection, enlarged lymph nodes, etc.[6].

Approaches to mitigate the dysfunctions above caused by excessive exercise include adjustment of training programmes, physiotherapy and some nutritional supplementation strategies.

Among newly emerging exploration, the cross-talk pathway between the gut microbiota and skeletal muscle has shown that alterations in microbiota composition are associated with muscle mass and function possibly via modification of fatty acid oxidation and muscle glycogen repletion [15], immune function [16], and oxidative properties [17]. Most notably, Mach and Fuster-Botella [18] suggested that the gut microbiome may play a key role in controlling oxidative stress and inflammatory responses by improving metabolism and energy expenditure during high-intensity exercise.

According to the review of Sire et al. [19] changes in the microbiota composition can affect muscle health in a variety of ways, including by influencing intestinal barrier strength, immune cell activation, and gutmuscle signaling pathways such as myostatin/activin signaling, IGF-1/PI3K/AKT/mTOR signaling, NF-KB signaling and FOXO signaling. Among, NF-KB belongs to the Rel subfamily, which is responsible for dimerization, recognition, binding to DNA, and interaction with inhibitory proteins [20]. By regulating inflammatory factors, enzymes and chemokines, it plays an important role in immunity, inflammation and apoptosis [21]. Through specific inflammation-related membrane proteins, the increase on expression of NF-KB is greatly mediated, such as the toll-like receptors (TLRs). Several researches have indicated that TLRs can mediate inflammatory responses via triggering the MyD88-dependent pathway, which promotes the activation of NF-KB evidenced by p-NF-KB levels [22]. Furthermore, the activation of NF-KB has been documented to play a vital role in the regulation of pro-inflammatory genes, including TNF-a [23].

A few of studies demonstrated the beneficial effects of a mixed probiotics in improving excessive exercise-induced stress. However, to date, we have a limited understanding the underlying signaling mechanism of a mixed probiotics in alleviating intestinal barrier dysfunction, skeletal muscle damage and inflammatory effect caused by excessive exercise. Some newly developed five probiotics strains, bifidobacterium lactis V9 isolated from the intestinal tract of healthy Chinese Mongolian children, lactobacillus casei Zhang (LCZ) and lactobacillus plantarum P-8 isolated from traditional Chinese Mongolian beverages, bifidobacterium lactis Probio-M8 and lactobacillus rhamnosus Probio-M9 isolated from the healthy Chinese Mongolian breast mild, had shown a good probiotic characteristics such as acid resistance, bile resistance, gastrointestinal colonization and health-improving effects.

Our study is to investigate the effects of complex probiotics supplementation on 8-wk excessive exerciseinduced skeletal muscle inflammation response and anti-oxidant status, and underlying mechanisms in rats.

\section{Materials And Methods}

\section{Bacteria strains}


The mixed probiotics $\left(4 \times 10^{11} \mathrm{CFU} / \mathrm{g}\right.$ ) composed of a matrix (maltodextrin) and five probiotic strains, bifidobacterium lactis V9, bifidobacterium lactis Probio-M8, lactobacillus rhamnosus Probio-M9, lactobacillus casei Zhang, lactobacillus plantarum P-8, and was donated by Professor Zhang Heping at Inner Mongolia Agricultural University. The lyophilized powder was suspended in distilled water and diluted to $4 \times 10^{9} \mathrm{CFU} / \mathrm{ml}$ when it was used daily. The control supplement contained of maltodextrin only.

\section{Animals}

Male mature Sprague Dawley rats (180-220 g weight) were purchased from Beijing Vital River Laboratory Animal Technology Co., Ltd. (Beijing, China). All the basal rat feed purchased from Beijing Keao Xieli Feed Co. Ltd. (Beijing, China) were replenished daily. Throughout the course of the study, rats were housed in plastic cages with free access to water and food and kept under similar conditions of climate, ventilation, temperature, humidity: $23-27^{\circ} \mathrm{C}, 50-70 \%$ relative humidity, and light/dark cycle of $12 \mathrm{~h} / 12 \mathrm{~h}$.

\section{Experimental design}

After one week acclimatization, rats were randomly distributed into four groups of 8 animals each. SC/EC and SP/EP groups received $1 \mathrm{ml}$ of the control supplement (maltodextrin only) and the mixed probiotics $\left(4.0 \times 10^{9} \mathrm{CFU} / \mathrm{ml}\right)$ by oral gavage separately 1 hour prior to the start of daily training for 8 weeks. A modified Hohl's treadmill protocol with incremental load [27] during 8-week treatment was used to establish the excessive exercise model.

\section{Sample collection and preparation}

Blood samples were collected under $10 \%$ chloral hydrate anesthesia $(0.3 \mathrm{ml} / 100 \mathrm{~g})$ into inert separate gel pro-coagulation tube by puncturing the abdominal aorta with disposable blood collection needle for medical purpose at 20-24 ${ }^{\text {th }}$ hour post the last exercise, and were left for $30 \mathrm{~min}$ at room temperature then centrifuged at $3000 \mathrm{rpm}$ for $15 \mathrm{~min}$ to separate the serum. Rat soleus was sampled after a complete cardiac arrested and rapidly rinsed with ice-cold isotonic saline, then snap-frozen in liquid nitrogen and stored in $-80^{\circ} \mathrm{C}$ refrigerator until analysis within 3 months. Serum was applied to assay CK, LDH, LPS levels, and soleus were used for the determination of T-AOC, GPx, CAT, SOD activities, LPS, MDA, TNF-a, IL-1 $\beta$, IL-6 levels, and TLR4, MyD88, p65, p-p65, Bax, Bcl2, caspase-9, caspase-3 protein relative expressions.

\section{Biochemical analysis}

Serum CK and LDH activities were determined by automatic biochemical analyzer (UniCel DxC 600, Beckman, USA). Commercially available kits were purchased from Beijing Leadman Biochemistry Co., Ltd. (Beijing, China).

\section{Preparation of muscle homogenates}


At a ratio of 1:10 (w/v), the soleus was homogenized in phosphate buffer solution (PBS, pH =7.4). After completely cracked by homogenizer (JK Ultra-Turrax T25, Germany), homogenates were centrifuged at $10,000 \mathrm{~g}$ for $15 \mathrm{~min}$ at $4^{\circ} \mathrm{C}$, and the resultant supernatant was used for the determination of muscle indicators. Total protein concentrations of the samples were determined using the bicinchonininc acid (BCA) protein assay kit (Invitrogen, USA).

\section{ELISA assay for LPS, IL-6, IL- $\beta$ and TNF- $\alpha$}

According to manufacturer's instructions, serum and soleus levels of LPS, serum IL- 6 and IL-1 $\beta$ and TNFa were measured using a sandwiched enzyme-linked immunosorbent assay (ELISA) kit (Shanghai Jianglai Biotech, Shanghai, China).

\section{Antioxidant status analysis}

The activities of GPX, CAT and superoxide dismutase (SOD) and levels of T-AOC and MDA in soleus were analyzed using the commercial kits in accordance with the manufacturer's instructions (Beyotime, China).

\section{Western blot analysis}

Frozen muscle tissues were weighed and homogenized in ice-cold lysis buffer containing protease and phosphatase inhibitor (Beyotime, China). Homogenates were centrifuged at 12,000 rpm for 20 min at 4 ${ }^{\circ} \mathrm{C}$, and the supernatants were immediately collected for use. Forty micrograms of protein were diluted in sample loading buffer and heated at $70^{\circ} \mathrm{C}$ for $10 \mathrm{~min}$. The denatured proteins were separated by sodium dodecyl sulphate-polyacrylamide gel electrophoresis (SDS-PAGE), and transferred onto polyvinylidene difluoride (PVDF) membranes, The membranes were blocked for $1 \mathrm{~h}$ at room temperature (RT) in Trisbuffered saline (TBS) containing 5\% BSA (for phosphorylated proteins) /nonfat dry milk (for nonphosphorylated proteins) and $0.1 \%$ Tween 20 (TBST), and then incubated overnight at $4{ }^{\circ} \mathrm{C}$ in TBST containing specific primary antibodies (anti-TLR4, anti-MyD88, anti-p65, anti-p-p65, anti-Bax, anti-Bcl2, anti-caspase-9, anti-caspase-3 or anti-GAPDH ). After washing with TBST buffer three times, the membranes were incubated with HRP-conjugated secondary antibody (1:4000, Invitrogen, USA) for $1 \mathrm{~h}$ at RT. The blots were visualized and analyzed by a Luminescent Image Analyzer (Tanon-5200, Tanon, China).

\section{Statistical analysis}

Results are expressed as mean \pm standard deviation (SD). A 2-way analysis of variance (ANOVA) was performed to determine the effect of exercise and probiotics followed by the LSD post hoc tests for multiple comparison in SPSS 20.0 (IBM, Armonk, NY, USA). Values of $p \leq 0.05$ were considered statistically significant.

\section{Results}


It was shown that excessive exercise demonstrated the elevated effects on serum CK and LDH activities in EC (Fig.1A) and in EP groups (Fig.1B) respectively compared to those in SC and in SP $(p<0.05)$.

\section{Effects of probiotics supplementation on LPS levels in serum and soleus}

As shown in Fig.2, Excessive exercise showed an increased effect on serum and soleus level of LPS in EC group compared with SC group $(p<0.01)$, and significantly decreased effect of probiotics supplementation on serum and soleus LPS level was seen in EP group, but not in SP group $(p<0.05)$.

\section{Effects of probiotics on inflammatory cytokines in soleus}

The results demonstrated that excessive exercise had a significant effect on IL-6, IL-1 $\beta$ and TNF-a levels $(p<0.01)$ (Fig.3). Probiotics usage alone did not influence these inflammatory factor levels in soleus (Fig.3, $p>0.05)$. EP group showed a significantly $(p<0.05)$ lower levels of IL-1 $\beta$ and TNF-a compared with EC group (Fig.3 B, C), but not IL-6 (Fig.3A, $p>0.05$ ).

\section{Effects of probiotics supplementation on the expression of TLR4 signaling factors in soleus}

Western blot analysis was performed to detect intramuscular protein expressions of TLR4, MyD88, p-NF$\mathrm{KB}$ and NF-KB involved in the downstream signaling pathway of LPS (Fig.4). There were no significant differences of TLRs/NF-KB signaling pathway expression between SC and SP groups (Fig.4, p囚0.05). Compared with SC group, the protein levels of TLR4 (Fig.4A, $p<0.01$ ), MyD88 (Fig.4B, $p<0.01$ ) in EC group were significantly increased, accompanied by an enhanced protein concentration of the p-NF-KB /NF-KB (Fig.4C, $p<0.01$ ). By contrast, activation of TLR4 (Fig.4A, $p<0.05$ ), MyD88 (Fig.4B, $p<0.05$ ) and p-NF-KB /NF-KB (Fig.4C, $p<0.05)$ in EP rats was reduced compared with EC group.

\section{Effects of probiotics supplementation on antioxidant enzymes and lipid peroxidation in soleus}

The T-AOC, GPx and CAT were significantly decreased $(p<0.05)$ in EC group compared with that of SC group (Fig.5A, C, D), and higher levels of T-AOC and GPx in EP group was seen compared to EC group (Fig.5A, C, $p<0.05$ ). There was no significant difference in SOD activity among the four groups (Fig.5B, $p$ $>0.05)$. MDA level was significantly $(p<0.05)$ increased in soleus of EC group compared to SC group, however, significantly reduced $(p<0.05)$ in EP group compared with that in EC group (Fig.5E).

\section{Effects of probiotics on cell apoptosis in soleus}

The results showed an upregulation of $\mathrm{Bax} / \mathrm{Bcl}-2$, cleaved caspase-9/procaspase-9 and cleaved caspase3 /procaspase-3 protein levels in EC group (Fig.6A-C, $p<0.05$ ), and declined tendency of those biomarkers was seen in EP group compared with those in EC group (Fig.6A-C, $p>0.05$ ).

\section{Discussion}

The overtraining-induced dysfunction is associated with both localized muscular disruptions and systemic physiological stress. 
Previous review has clarified an increase in serum CK, LDH, TNF- $a$ and IL-1 $\beta$ in rats submitted to progressive resistance exercise $\left[{ }^{28}\right]$. The overtraining leads to an oxidative stress $\left.{ }^{29}\right]$ as demonstrated in one animal study, which overtraining induced by downhill running can trigger oxidative stress responses in skeletal muscle cells and whole blood [ ${ }^{30}$ ]. In addition, it has been proposed that cells enter a state of apoptosis after exposure to high levels of ROS [ $\left.{ }^{31}\right]$. ROS serves as a second messenger in the cell signal transduction pathway by activating proteolytic systems including calpain protease and caspase, and ultimately leading to muscle atrophy and muscle strength decline $\left.{ }^{[2}\right]$. It was reported that maximum swimming led to lipid peroxidation, NF-KB activation in skeletal muscle and increased plasma IL-6 level [33].

A symposium report suggested that the benefits of probiotics consumption might be related to their gutmodulating properties, for instance, metabolic modulation, immunomodulatory, anti-inflammatory capability, anti-oxidant effect, etc. $\left.{ }^{[34}{ }^{35}\right]$. A pioneering study $\left.{ }^{36}\right]$ showed that a daily treatment with Lactobacillus plantarum TWK10 (LP10) induced a dose-dependently reduction in serum lactic acid and ammonia levels and CK activity which are typically increased in fatigue and muscle damage condition with highly intensive endurance exercise, and an improvement of skeletal muscle relative mass and endurance performance in mice.

Being consistent with previous investigations, our analysis in vivo showed that excessive exercise led to an increase in serum CK and LDH activities, which should enough reflect an exercise-induced skeletal muscle micro-damage following 8-week incremental load training (Fig.1). Otherwise, increased levels of pro-inflammatory cytokines (IL-6, IL-1 $\beta$ and TNF-a) (Fig.3), MDA (Fig.5) and decreased level of soleus antioxidant levels (T-AOC, GPx and CAT) also suggested that excessive exercise-induced injury model was established successfully. Although, in our study, probiotics supplementation failed to reduce serum CK and LDH activities to a significant extent in all exercise-involved groups like SP and EP compared to SC and SP respectively (Fig.1), we saw the anti-inflammatory effect of probiotics supplementation, which implied the potential anti-inflammatory mechanism of probiotics supplementation that Chen et al. had speculated the underlying mechanism of probiotics (LP10) supplementation in reducing proinflammatory cytokines related to the improvements in skeletal muscle atrophy markers. On the other hand, the mixed probiotics supplementation demonstrated antioxidant- and immune-improving effects in according with some of studies.

At present, sufficient evidences exist to support the contention that strenuous exercise is linked to an overwhelming damage of gut barrier resulting in endotoxin translocation, pro-inflammatory cytokine production, and impaired nutrient absorption [ $\left.{ }^{37}{ }^{38}\right]$. Alteration in gut-microbiota homeostasis is associated with facilitated passage of LPS from intestinal barrier to systemic circulation. Accordingly, the cumulative LPS is considered to be an useful marker of intestinal barrier function. To determine the impact of the excessive exercise and probiotics supplementation on intestinal barrier, we measured LPS levels both in serum and in skeletal muscle. Earlier, it was confirmed in a human study that a significant increase in LPS concentration could be considered endotoxemia in $68 \%$ of athletes one hour after a long- 
distance triathlon $\left.{ }^{39}\right]$. Consistent with the research, our results clearly showed that excessive exercise as a negative factor upregulated LPS levels in blood and skeletal muscle. By modulating the imbalance of intestinal flora and protecting the mucosal barrier, mixed probiotics supplementation prevented the passage of LPS from intestinal barrier to systemic circulation, and skeletal muscle ultimately.

In addition, early research had revealed that the leakage of luminal LPS from gram-negative bacteria plays a pivotal role in initiating low-grade inflammation [ ${ }^{40}$ ]. The LPS is bound to TLR4, consequently, leads to interaction with its downstream adaptor protein MyD88 and activated NF-KB translocated into the cell nucleus and accelerated NF-KB phosphorylation, then combines to promoter or enhancer binding sites in downstream genes that is responsible for the inflammatory response, induces the high expressions of pro-inflammatory cytokines such as TNF-a, IL-1 $3\left[{ }^{41}\right]$ as we saw in this study (Fig. 3). Furthermore, NF-KB can be activated by its feedback, which amplifies the inflammatory response and manifests as accumulation of pro-inflammatory cytokine, consequently up-regulates the expression of $\mathrm{NF}-\mathrm{KB}$ and induces apoptosis $\left[{ }^{42}\right]$. A previous study has noted that accompanied by an attenuation of the TLR4/NF-KB signaling pathway, the pretreatment with diclofenac decreased the TNF-a, IL-6 and IL-1 $\beta$ mRNA expressions induced by acute exercise in rat skeletal muscle $\left[{ }^{43}\right]$.

Probiotic strains have been shown to stimulate dendritic cell-induced T-regulatory cells to attenuate IL-6 and TNF-a production [ $\left.{ }^{44}\right]$. Previous studies have confirmed that administration of LcZ (lactobacillus casei Zhang) to rats treated with LPS/D - GalN (D-galactosamine) suggested that TLR4 mRNA and protein levels I-KB phosphorylation and NF-KB translocation in liver cells were significantly reduced $\left.{ }^{45}\right]$. The latest research results indicated that mixed probiotics treatment significantly reduced the level of muscle LPS contributed to downregulating the TLR4/NF-KB signalling pathway in intestinal tissue, and consequently markedly alleviated the production of pro-inflammatory factors (IL- $1 \beta$ and TNF- $\alpha$ ) $\left[{ }^{46}\right]$. It is consistent with previous research, this study confirmed that administration of complex probiotics remarkably down-regulated the TLRs/NF-kB signaling pathway by weakening the expression of TLR4 and MyD88 and inhibiting NF-KB nuclear translocation, which is consistent with changing trend of LPS level in soleus muscle (Fig. 4). Furthermore, probiotics attenuated overtraining-induced inflammatory response via significantly reducing the levels of pro-inflammatory factors (TNF- $a$ and IL-1 $\beta$ ) due to the suppression of the TLR4/NF-KB signaling pathway.

Some probiotic strains could elicit anti-oxidant effect in protecting intestinal cells from the harmful effect of free radicals $\left[{ }^{47}\right]$ through actions of manganese superoxide dismutase, pseudo-catalase and peroxidase enzymes. Bifidobacterium ATCC 29521, for example, was found to be a potential biogenic antioxidant that could be used in microbiology and food industry $\left[{ }^{48}\right]$. Currently, except for various antioxidative effect of probiotics well carried out in vitro, the beneficial effect of probiotic supplementation on oxidative stress have also been in vivo demonstrated. MDA is frequently used as a marker for free radical damage. A study by Badehnoosh et al. $\left[{ }^{49}\right]$ indicated that oral administration of mixed probiotics capsule containing lactobacillus acidophilus, lactobacillus casei and bifidobacterium for 6 weeks resulted in a decreased plasma MDA concentration and a significantly increased T-AOC level in pregnant women with 
gestated diabetes. Martarelli et al. $\left[{ }^{50}\right]$ have suggested that athletes suffering from oxidative stress might benefit from the supplementation of Lactobacillus rhamnosus (LGG) as a result of improving antioxidant levels and the ability to neutralize ROS. The current results showed that the administration of complex probiotics obviously ameliorated decreased T-AOC level and GPx activity in skeletal muscle of rats challenged with long-term increasingly intensive exercise (Fig.5), which indicates that certain probiotics may be used in attenuating oxidative stress and better restoration of muscle damage caused by excessive exercise.

Anti-oxidative effect of probiotics may be an important mechanism involved in its function such as antiinflammatory response because both local and systemic inflammatory responses are associated with the production of ROS. In line with the review by LeBlanc et al. [ $\left.{ }^{51}\right]$, which indicated antioxidant enzymes (such as CAT and SOD) produced by lactic acid bacteria could eliminate ROS and modulate the release of host cytokines (such as IL-10), and alleviate the severity of intestinal inflammatory diseases. Furthermore, a study confirmed that L. plantarum C88 could block alcohol-induced chronic and acute liver damage by improving intestinal barrier dysfunction, inhibiting intestinal endotoxin-mediated inflammation, and ameliorating oxidative stress [ $\left.{ }^{52}\right]$. Additionally, elevated synthesis and secretion of pro-inflammatory cytokines such as IL- 6 or TNF- $a$ caused by intense exercise induced protein degradation and cell apoptosis, inhibited protein synthesis as well as impaired the metabolic and contractile properties of skeletal muscles, which are considered to be one of causes of muscle atrophy [ $\left.{ }^{53}{ }^{54}\right]$. Therefore, probiotics might be conducive to the function of anti-inflammation due to the alteration in antioxidant levels. This hypothesis was confirmed in our study, which showed that the supplementation with mixed probiotics reduced MDA levels and increased T-AOC level and GPx activity (Fig.5), synchronously declined pro-inflammatory cytokines, TNF-a and IL-1 $\beta$ levels, in skeletal muscle of overtraining rats (Fig.3).

Generally, Bcl-2 is regarded as an anti-apoptotic protein. Moreover, activated Bax leads to subsequent activation of caspases by releasing cytochrome $\mathrm{C}$ to the outside of mitochondria. As pro-apoptotic proteins, caspases are widely expressed in most cells, which could amplify the apoptotic signaling pathway and accelerate cell death. It has previously been confirmed that accompanied with downregulated the ratio of $\mathrm{Bax} / \mathrm{Bcl}-2$ and increased the expression of procaspase-3 while reducing the level of cleaved caspase-3, prophylactic treatment with L. helveticus R0052 + B. longum R0175 alleviated hippocampal apoptosis induced by LPS in Wistar rats [55]. Another study investigated the action of $L$. plantarum C88 in preventing AFB1-induced liver injury via inhibiting the secretion of pro-inflammatory cytokines (IL-1 $\beta$, IL- 6 and TNF-a), impeding NF-KB nuclear translocation, and reducing the levels of Bax and caspase-3, elevating the level of $\mathrm{Bcl}-2$, which ameliorated the excessive apoptosis in liver $\left[{ }^{56}\right]$. In the current study, changes of apoptotic protein expression of Bax, Bcl-2, caspase-9 and caspase-3 indicated the occurrence of apoptosis after the excessive exercise. However, the results shown only a declined tendency of apoptotic factors after the utility of probiotics supplementation. Further study should be conducted in the future to determine the beneficial effect of this mixed probiotics on ameliorating cell apoptosis in skeletal muscle. 


\section{Conclusions}

These serum and intramuscular indicators suggest that effects of complex probiotics supplementation used in the research should protect against overtraining-induced muscle micro damage in rats via protecting mucosal barrier, preventing LPS from entering into systemic circulation, inhibiting NF-KBmediated inflammatory responses, and improving antioxidant status.

Our results indicate a role for the microbiota in intestinal tract as a key nutritional interventive target for exercise-induced muscle dysfunction. Administration of complex probiotics may be one of healthy nutritional approaches for athletes.

\section{Declarations}

\section{Ethical Approval}

This study was approved by Ethical Commission at National Institute of Sports Medicine, China.

\section{Availability of supporting data}

The datasets used or analysed during the current study are available from the corresponding author on request.

\section{Consent for publication}

Not applicable.

\section{Competing interests}

The authors declare that they have no conflict of interest and that the results of the present study do not constitute endorsement by JISSN.

\section{Funding}

This article is funded by Key Projects (2016YFD0400603) in the National Science and Technology Program during the Thirteen Five-year Plan Period. There is no financial support received from any manufacturers in this study.

\section{Authors' contributions}

$\mathrm{XY}$ was responsible for study design, animal training and sampling, data collection, statistical analysis and drafting the article. SJ, WH, TQ and HQ joined animal training, sampling and data collection. YM were responsible for study design, revising the article and final approval of the version to be published.

\section{Acknowledgements}


The authors thank Prof. Zhang Heping and his teams at China Inner Mongolia Agricultural University for the gift of complex probiotics preparations. All authors read and approved the final manuscript for publication.

\section{Authors' information}

Xia Yali, MSc

Email:1977299033@qq.com

Shao Jing $\mathbb{P} h . \mathrm{D}$

Email『shaojing-rcees@126.com

Wang Hui, Ph.D candidate

Email:wanghui_0@sina.cn

Tan Qiushi囚B.S.

Email: qiushitan@126.com

Han Qi, MSc

Email: hanqi0418@163.com

Yi Muqing, Ph.D, MD

Email: muqingyi@163.com

\section{References}

1. Parnell JA, Wagner-Jones $K$, Madden RF, Erdman KA. Dietary restrictions in endurance runners to mitigate exercise-induced gastrointestinal symptoms. J Int Soc Sports Nutr. 2020;17.

2. Hotfiel T, Mayer I, Huettel M, Hoppe MW, Engelhardt M, Lutter C, et al. Accelerating Recovery from Exercise-Induced Muscle Injuries in Triathletes: Considerations for Olympic Distance Races. Sports. 2019;7:143.

3. Suzuki K, Tominaga T, Ruhee RT, Ma S. Characterization and modulation of systemic inflammatory response to exhaustive exercise in relation to oxidative stress. Antioxidants. 2020;9.

4. Juszkiewicz A, Glapa A, Basta P, Petriczko E, Żołnowski K, Machaliński B, et al. The effect of Ltheanine supplementation on the immune system of athletes exposed to strenuous physical exercise. J Int Soc Sports Nutr. 2019;16.

5. Meeusen R, Duclos M, Gleeson M, Rietjens G, Steinacker J, Urhausen A. Prevention, diagnosis and treatment of the Overtraining Syndrome. ECSS position statement "task force." Eur J Sport Sci. 
2006;6:1-14.

6. Meeusen R, Duclos M, Foster C, Fry A, Gleeson M, Nieman D, et al. Prevention, diagnosis, and treatment of the overtraining syndrome: Joint consensus statement of the european college of sport science and the American College of Sports Medicine. Med Sci Sports Exerc. 2013;45:186-205.

7. FAO/WHO. Guidelines for the evaluation of probiotics in food (Working Group on Drafting Guidelines for the Evaluation of Probiotics in Food). Food Agric Organ United Nations World Heal Organ [Internet]. 2002;21:1-11.

8. Karczewski J, Troost FJ, Konings I, Dekker J, Kleerebezem M, Brummer R-JM, et al. Regulation of human epithelial tight junction proteins by Lactobacillus plantarum in vivo and protective effects on EPithelial barrier. American journal of physiology. Gastrointestinal and liver physiology,. Am J Physiol Gastrointest Liver Physiol [Internet]. 2010;298:G851-9.

9. 9Michalickova DM, Kostic-Vucicevic MM, Vukasinovic-Vesic MD, Stojmenovic TB, Dikic N V., Andjelkovic MS, et al. Lactobacillus helveticus Lafti L10 Supplementation Modulates Mucosal and Humoral Immunity in Elite Athletes. J Strength Cond Res. 2017;31:62-70.

10. Borchers AT, Selmi C, Meyers FJ, Keen CL, Gershwin ME. Probiotics and immunity. J. Gastroenterol. 2009. p. 26-46.

11. Strasser B, Geiger D, Schauer M, Gostner JM, Gatterer H, Burtscher M, et al. Probiotic supplements beneficially affect tryptophan-kynurenine metabolism and reduce the incidence of upper respiratory tract infections in trained athletes: A randomized, double-blinded, placebo-controlled trial. Nutrients. 2016;8:752.

12. Carabotti M, Scirocco A, Maselli MA, Severi C. The gut-brain axis: Interactions between enteric microbiota, central and enteric nervous systems. Ann Gastroenterol. 2015;28:203-9.

13. Vajro P, Paolella G, Fasano A. Microbiota and gut-liver axis: Their influences on obesity and obesityrelated liver disease. J. Pediatr. Gastroenterol. Nutr. 2013. p. 461-8.

14. Tang WHW, Kitai T, Hazen SL. Gut microbiota in cardiovascular health and disease. Circ. Res. 2017. p. 1183-96.

15. Fushimi T, Tayama K, Fukaya M, Kitakoshi K, Nakai N, Tsukamoto Y, et al. The efficacy of acetic acid for glycogen repletion in rat skeletal muscle after exercise. Int J Sports Med. 2002;23:218-22.

16. Ticinesi A, Nouvenne A, Cerundolo N, Catania P, Prati B, Tana C, et al. Gut microbiota, muscle mass and function in aging: A focus on physical frailty and sarcopenia. Nutrients. 2019;11.

17. Pan JH, Kim JH, Kim HM, Lee ES, Shin DH, Kim S, et al. Acetic acid enhances endurance capacity of exercise-trained mice by increasing skeletal muscle oxidative properties. Biosci Biotechnol Biochem. 2015;79:1535-41.

18. Mach N, Fuster-Botella D. Endurance exercise and gut microbiota: A review. J. Sport Heal. Sci. 2017. p. 179-97.

19. De Sire R, Rizzatti G, Ingravalle F, Pizzoferrato M, Petito V, Lopetuso L, et al. Skeletal muscle-gut axis: Emerging mechanisms of sarcopenia for intestinal and extra intestinal diseases. Minerva Gastroenterol. Dietol. 2018. p. 351-62. 
20. Oliveira-Marques V, Marinho HS, Cyrne L, Antunes F. Role of hydrogen peroxide in NF-KB activation: From inducer to modulator. Antioxidants Redox Signal. 2009. p. 2223-43.

21. Lawrence T, Willoughby DA, Gilroy DW. Anti-inflammatory lipid mediators and insights into the resolution of inflammation. Nat. Rev. Immunol. 2002. p. 787-95.

22. Imler JL, Hoffmann JA. Toll receptors in innate immunity. Trends Cell Biol. 2001. p. 304-11.

23. Oeckinghaus A, Hayden MS, Ghosh S. Crosstalk in NF-kB signaling pathways. Nat. Immunol. 2011. p. 695-708.

24. Georges J, Lowery RP, Yaman G, Kerio C, Ormes J, McCleary SA, et al. The effects of probiotic supplementation on lean body mass, strength, and power, and health indicators in resistance trained males: A pilot study. J Int Soc Sports Nutr. 2014;11.

25. Jäger R, Shields K, Sharp M, Partl J, Wilson JM, Lowery RP, et al. Effects of probiotic supplementation on markers of skeletal muscle damage, perceived recovery and athletic performance after an intense single leg training bout. J Int Soc Sports Nutr. 2015;12.

26. Jäger R, Purpura M, Stone JD, Turner SM, Anzalone AJ, Eimerbrink MJ, et al. Probiotic Streptococcus thermophilus FP4 and Bifidobacterium breve BR03 supplementation attenuates performance and range-of-motion decrements following muscle damaging exercise. Nutrients. 2016;8.

27. Hohl R, Ferraresso RLP, De Oliveira RB, Lucco R, Brenzikofer R, De Macedo DV. Development and characterization of an overtraining animal model. Med Sci Sports Exerc. 2009;41:1155-63.

28. Raizel R, Leite JSM, Hypólito TM, Coqueiro AY, Newsholme P, Cruzat VF, et al. Determination of the anti-inflammatory and cytoprotective effects of I-glutamine and I-alanine, or dipeptide, supplementation in rats submitted to resistance exercise. Br J Nutr. 2016;116:470-9.

29. Tanskanen M, Atalay M, Uusitalo A. Altered oxidative stress in overtrained athletes. J Sports Sci. 2010;28:309-17.

30. Pereira BC, Pauli JR, Antunes LMG, De Freitas EC, De Almeida MR, De Paula Venâncio V, et al. Overtraining is associated with DNA damage in blood and skeletal muscle cells of Swiss mice. BMC Physiol. 2013;13.

31. Cao C, Lu S, Kivlin R, Wallin B, Card E, Bagdasarian A, et al. AMP-activated protein kinase contributes to UV- and H2O 2-induced apoptosis in human skin keratinocytes. J Biol Chem. 2008;283:28897-908.

32. Powers SK, Jackson MJ. Exercise-induced oxidative stress: Cellular mechanisms and impact on muscle force production. Physiol. Rev. 2008. p. 1243-76.

33. Cleto LS, Oleto AF, Sousa LP, Barreto TO, Cruz JS, Penaforte CL, et al. Plasma cytokine response, lipid peroxidation and NF-KB activation in skeletal muscle following maximumprogressive swimming. Brazilian J Med Biol Res. 2011;44:546-52.

34. Thomas L V., Suzuki K, Zhao J. Probiotics: A proactive approach to health. A symposium report. Br J Nutr. 2015. p. S1-15.

35. Wang Y, Wu Y, Wang Y, Xu H, Mei X, Yu D, et al. Antioxidant properties of probiotic bacteria. Nutrients. 2017. 
36. Chen YM, Wei L, Chiu YS, Hsu YJ, Tsai TY, Wang MF, et al. Lactobacillus plantarum TWK10 supplementation improves exercise performance and increases muscle mass in mice. Nutrients. $2016 ; 8$.

37. van Wijck K, Lenaerts K, van Loon LJC, Peters WHM, Buurman WA, Dejong CHC. Exercise-Induced splanchnic hypoperfusion results in gut dysfunction in healthy men. PLoS One. 2011;6.

38. van Wijck K, Pennings B, van Bijnen AA, Senden JMG, Buurman WA, Dejong CHC, et al. Dietary protein digestion and absorption are impaired during acute postexercise recovery in young men. Am J Physiol - Regul Integr Comp Physiol. 2013;304.

39. Jeukendrup AE, Vet-Joop K, Sturk A, Stegen JHJC, Senden J, Saris WHM, et al. Relationship between gastro-intestinal complaints and endotoxaemia, cytokine release and the acute-phase reaction during and after a long-distance triathlon in highly trained men. Clin Sci. 2000;98:47-55.

40. Cani PD, Amar J, Iglesias MA, Poggi M, Knauf C, Bastelica D, et al. Metabolic endotoxemia initiates obesity and insulin resistance. Diabetes. 2007;56:1761-72.

41. Checker R, Patwardhan RS, Sharma D, Menon J, Thoh M, Bhilwade HN, et al. Schisandrin B exhibits anti-inflammatory activity through modulation of the redox-sensitive transcription factors Nrf2 and NF-kB. Free Radic Biol Med. 2012;53:1421-30.

42. Yeom M, Kim JH, Min JH, Hwang MK, Jung HS, Sohn Y. Xanthii fructus inhibits inflammatory responses in LPS-stimulated RAW 264.7 macrophages through suppressing NF-KB and JNK/p38 MAPK. J Ethnopharmacol [Internet]. Elsevier; 2015;176:394-401.

43. Barcelos RP, Bresciani G, Cuevas MJ, Martínez-Flórez S, Soares FAA, González-Gallego J. Diclofenac pretreatment modulates exercise-induced inflammation in skeletal muscle of rats through the TLR4/NF-kB pathway. Appl Physiol Nutr Metab. 2017;42:757-64.

44. Groeger D, O’Mahony L, Murphy EF, Bourke JF, Dinan TG, Kiely B, et al. Bifidobacterium infantis 35624 modulates host inflammatory processes beyond the gut. Gut Microbes. 2013;4:325-39.

45. Wang Y, Li Y, Xie J, Zhang Y, Wang J, Sun X, et al. Protective effects of probiotic Lactobacillus casei Zhang against endotoxin- and d-galactosamine-induced liver injury in rats via anti-oxidative and antiinflammatory capacities. Int Immunopharmacol. 2013;15:30-7.

46. Deng X, Zheng C, Wang S, Yang R, Liu Z, Chen T. Treatment with a probiotic combination reduces abdominal adhesion in rats by decreasing intestinal inflammation and restoring microbial composition. Oncol Rep. 2020;43:986-98.

47. Calderini E, Celebioglu HU, Villarroel J, Jacobsen S, Svensson B, Pessione E. Comparative proteomics of oxidative stress response of Lactobacillus acidophilus NCFM reveals effects on DNA repair and cysteine de novo synthesis. Proteomics. 2017;17.

48. Wang BG, Xu HB, Xu F, Zeng ZL, Wei H. Efficacy of oral bifidobacterium bifidum ATCC 29521 on microflora and antioxidant in mice. Can J Microbiol. 2015;62:249-62.

49. Badehnoosh B, Karamali M, Zarrati M, Jamilian M, Bahmani F, Tajabadi-Ebrahimi M, et al. The effects of probiotic supplementation on biomarkers of inflammation, oxidative stress and pregnancy outcomes in gestational diabetes. J Matern Neonatal Med. 2018;31:1128-36. 
50. Martarelli D, Verdenelli MC, Scuri S, Cocchioni M, Silvi S, Cecchini C, et al. Effect of a probiotic intake on oxidant and antioxidant parameters in plasma of athletes during intense exercise training. Curr Microbiol. 2011;62:1689-96.

51. LeBlanc J, Carmen S, Turk M, Lima F, Pontes D, Miyoshi A, et al. Mechanisms Involved in the AntiInflammatory Properties of Native and Genetically Engineered Lactic Acid Bacteria. Anti-Infective Agents. 2013;11:59-69.

52. Zhao L, Jiang Y, Ni Y, Zhang T, Duan C, Huang C, et al. Protective effects of Lactobacillus plantarum C88 on chronic ethanol-induced liver injury in mice. J Funct Foods. 2017;35:97-104.

53. Tisdale MJ. Mechanisms of cancer cachexia. Physiol. Rev. 2009. p. 381-410.

54. Grosicki GJ, Fielding RA, Lustgarten MS. Gut Microbiota Contribute to Age-Related Changes in Skeletal Muscle Size, Composition, and Function: Biological Basis for a Gut-Muscle Axis. Calcif. Tissue Int. 2018. p. 433-42.

55. Mohammadi G, Dargahi L, Naserpour T, Mirzanejad Y, Alizadeh SA, Peymani A, et al. Probiotic mixture of Lactobacillus helveticus R0052 and Bifidobacterium longum R0175 attenuates hippocampal apoptosis induced by lipopolysaccharide in rats. Int Microbiol. 2019;22:317-23.

56. Huang L, Zhao Z, Duan C, Wang C, Zhao Y, Yang G, et al. Lactobacillus plantarum C88 protects against aflatoxin B1-induced liver injury in mice via inhibition of NF-kB-mediated inflammatory responses and excessive apoptosis. BMC Microbiol. 2019;19.

\section{Figures}

\section{(A) Serum CK activity}

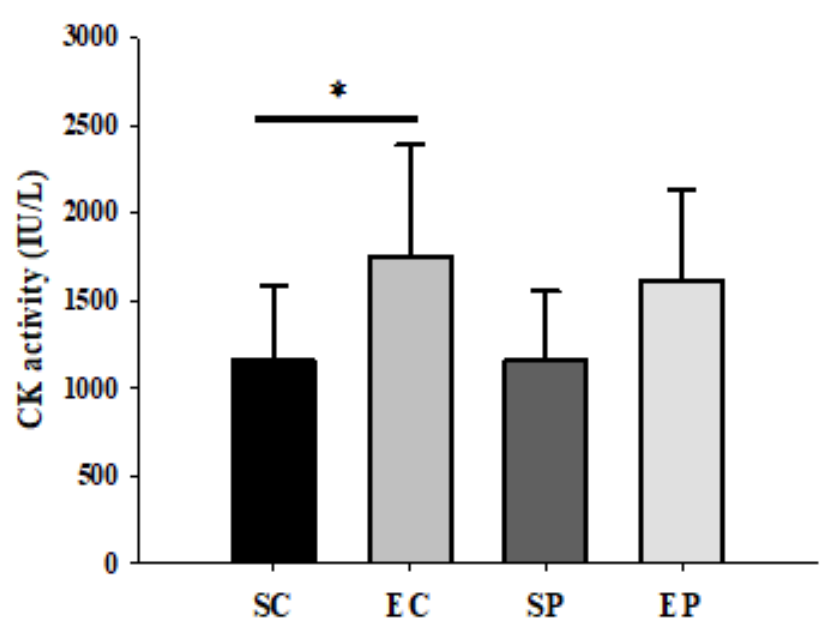

(B) Serum LDH avtivity

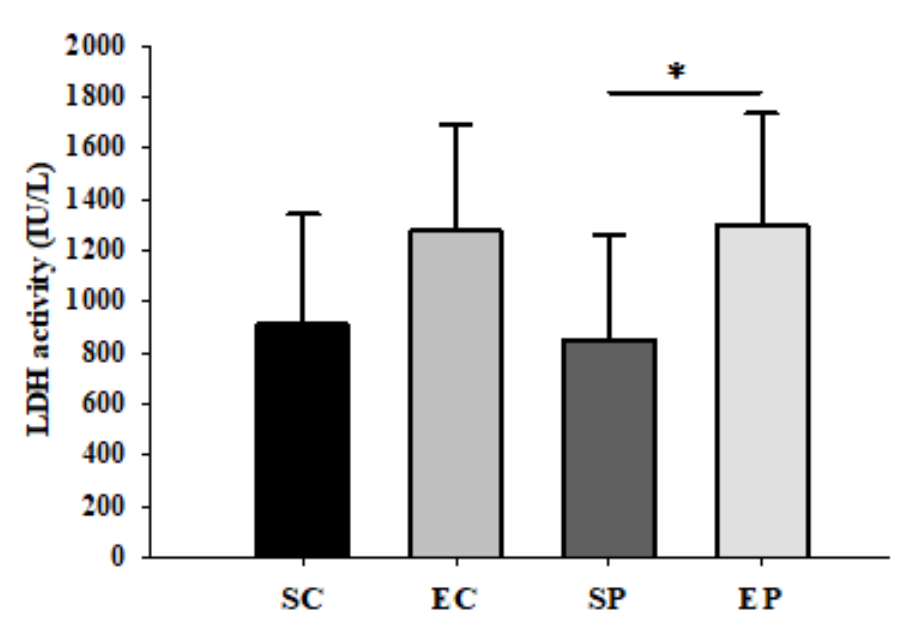

Figure 1

Effects of excessive exercise on rat serum CK (A) and LDH (B) activities. ${ }^{*} \mathrm{p} \leq 0.05$. 
(A) Serum LPS level

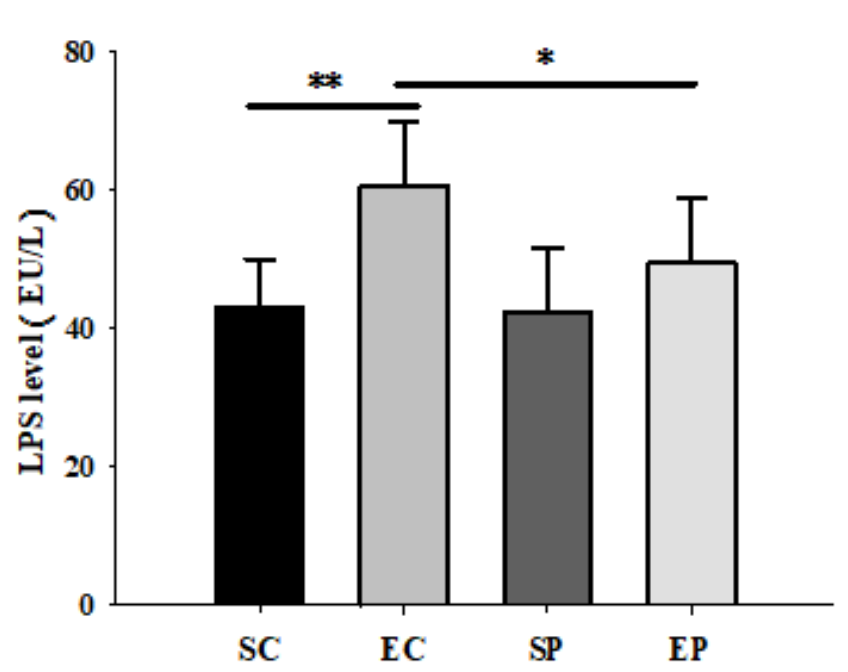

(B) Soleus LPS level

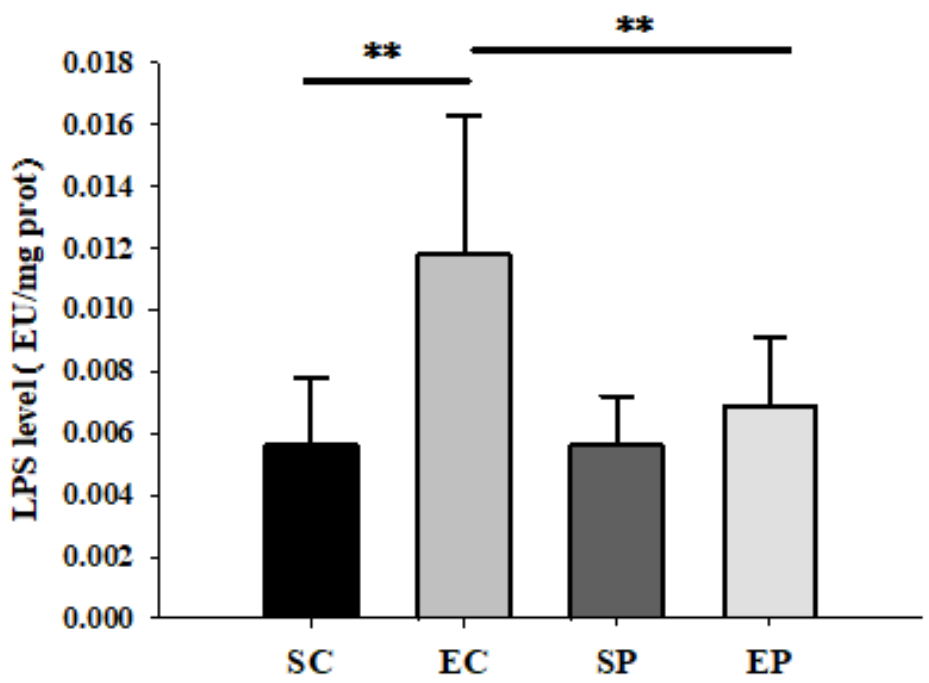

Figure 2

Effects of excessive exercise and probiotics supplementation on levels of LPS in serum (A) and soleus (B). ${ }^{\star} p \leq 0.05,{ }^{\star \star} p \leq 0.01$. 
(A) Soleus IL-6 level

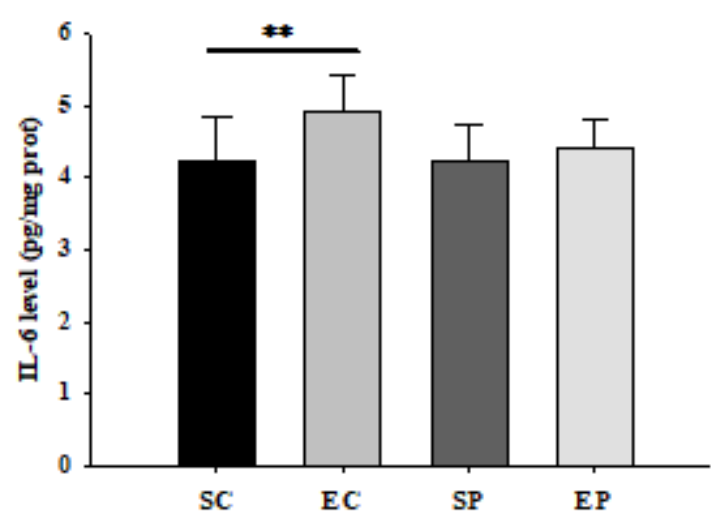

(C) Soleus TNF- $\alpha$ level

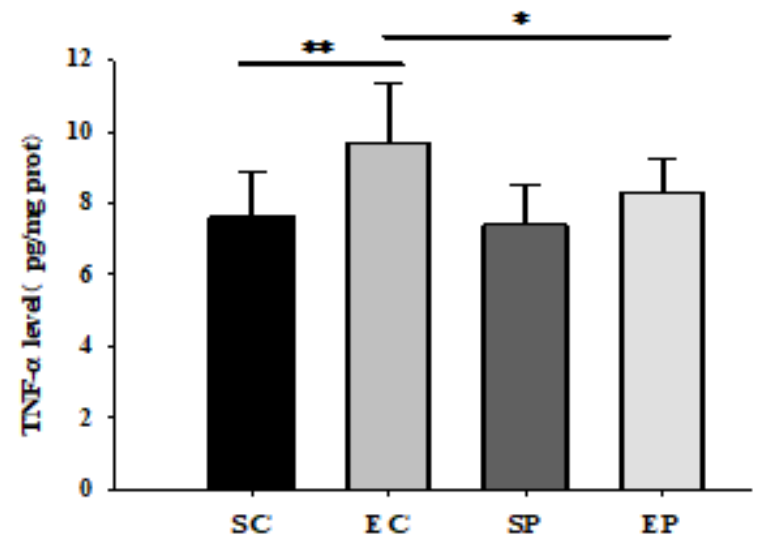

(B) Soleus IL-1 $\beta$ lev el

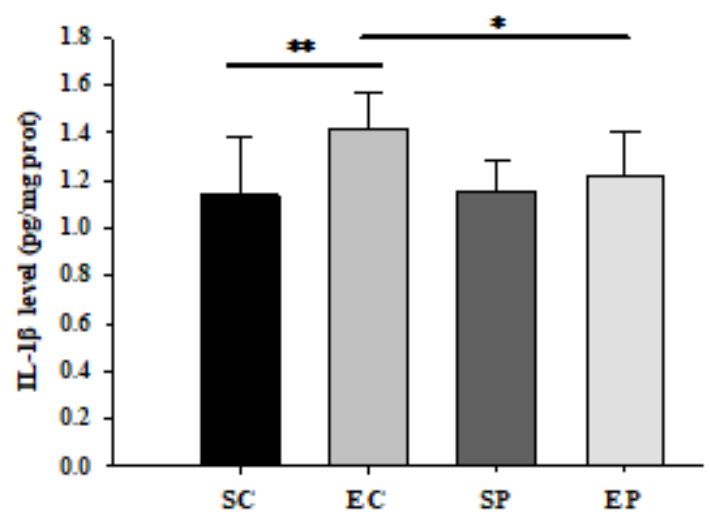

Figure 3

Effects of excessive exercise and probiotics supplementation on levels of IL-6 (A), IL-1 (B) and TNF- $\alpha$ (C) in soleus. ${ }^{*} \mathrm{p} \leq 0.05,{ }^{\star *} \mathrm{p} \leq 0.01$. 
(A)

Soleus TLR4 lev el

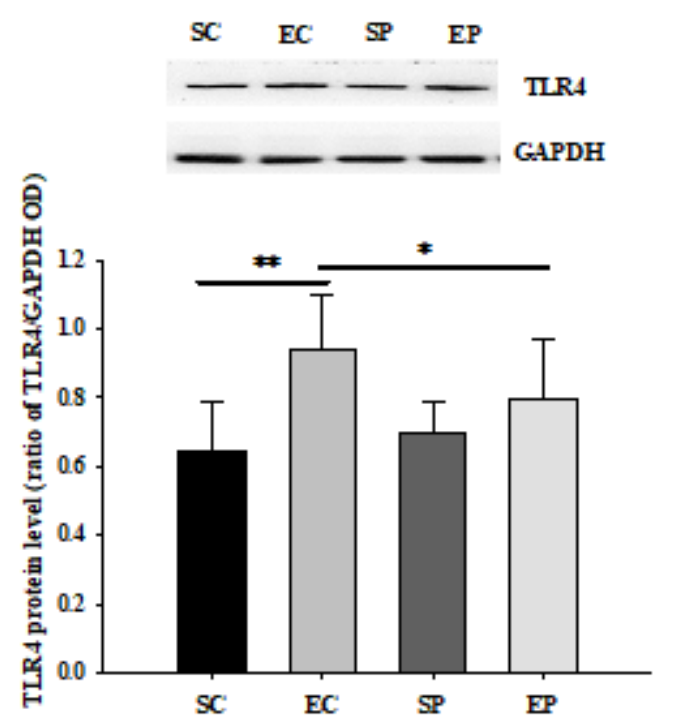

(C)

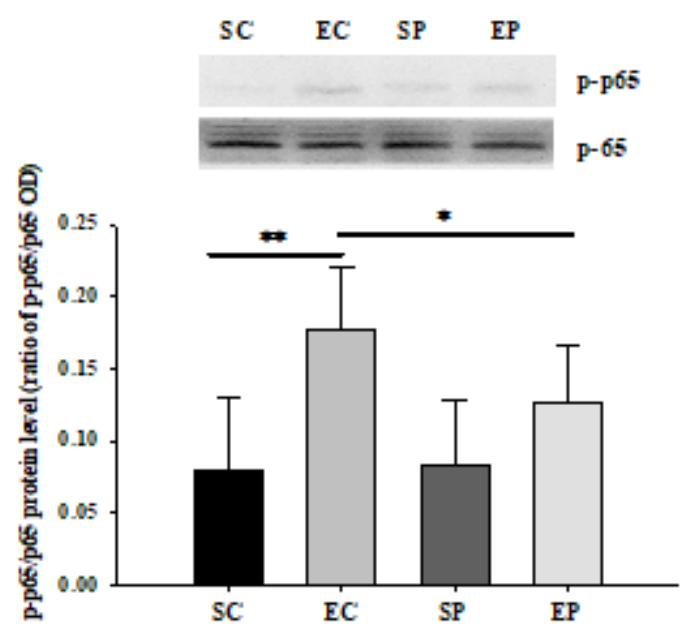

(B) Soleus MyD88 level

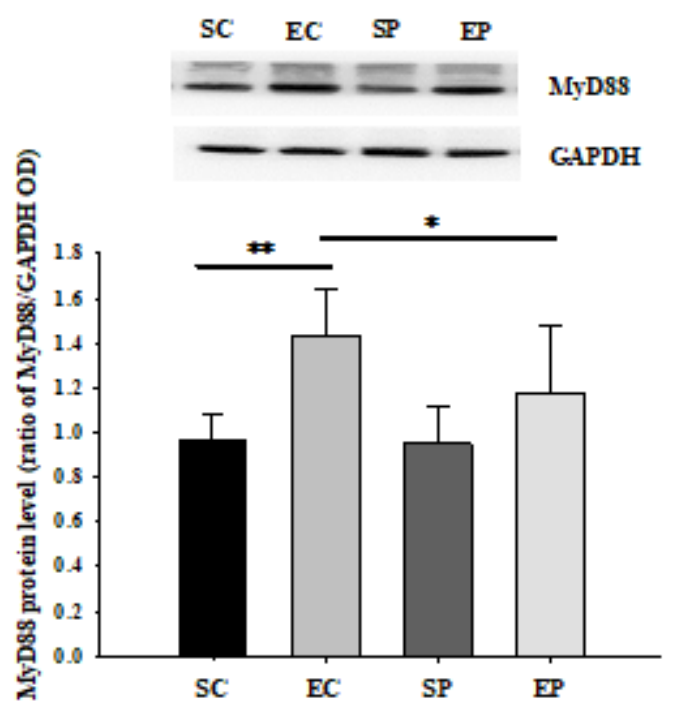

Figure 4

Effects of excessive exercise and probiotics supplementation on protein content of TLR4 (A), MyD88 (B) and $p-p 65 / p 65(C)$ in soleus. ${ }^{*} p \leq 0.05,{ }^{*} p \leq 0.01$. 
(A) Soleus T-AOC lerel

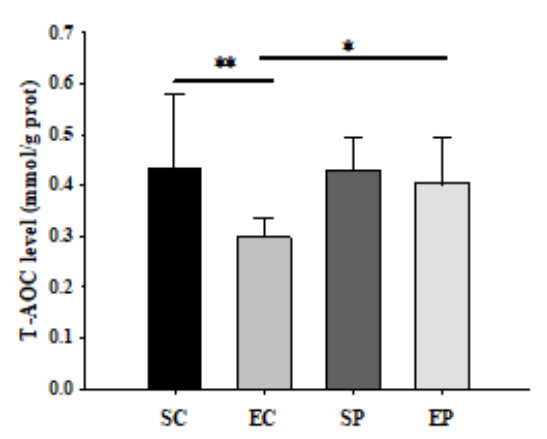

(C) Soleus GPx activity

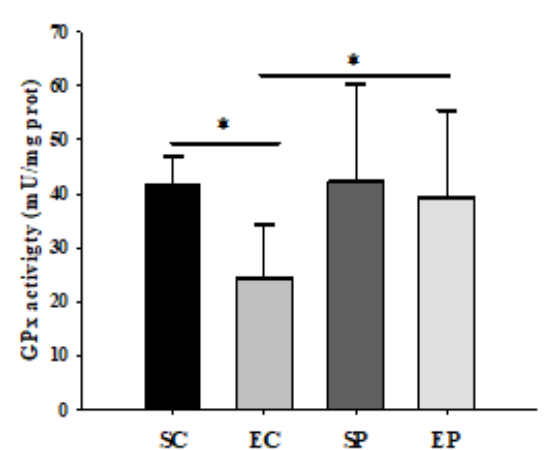

(E) soleus NDA level

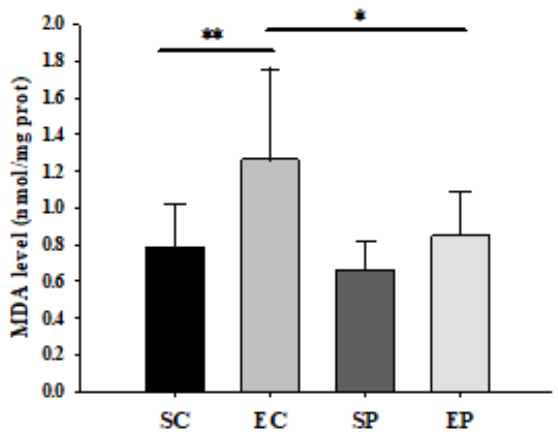

(B) Soleus SOD activity

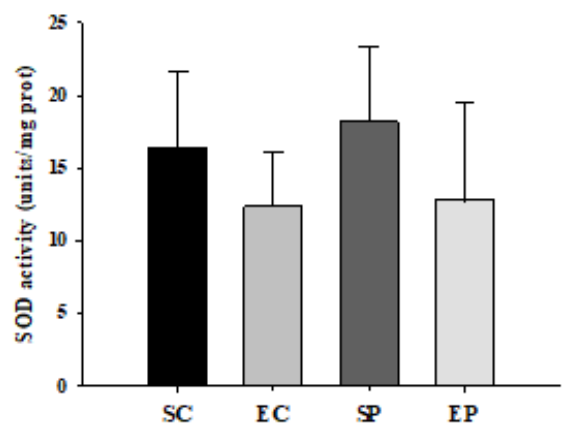

(D)

Soleus CAT activ ity

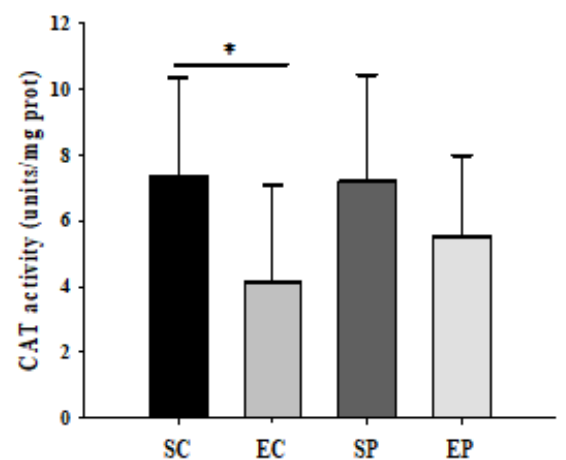


(A)

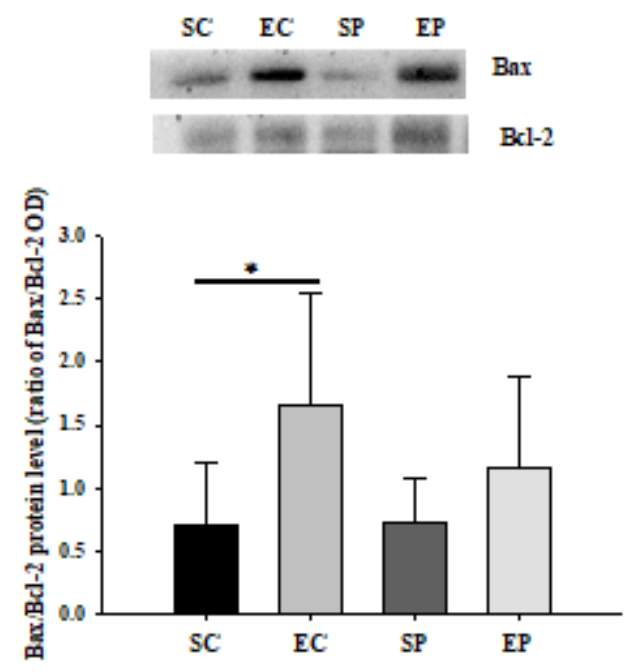

(C) Soleus cleaved caspase-3/procaspase-3 lev el

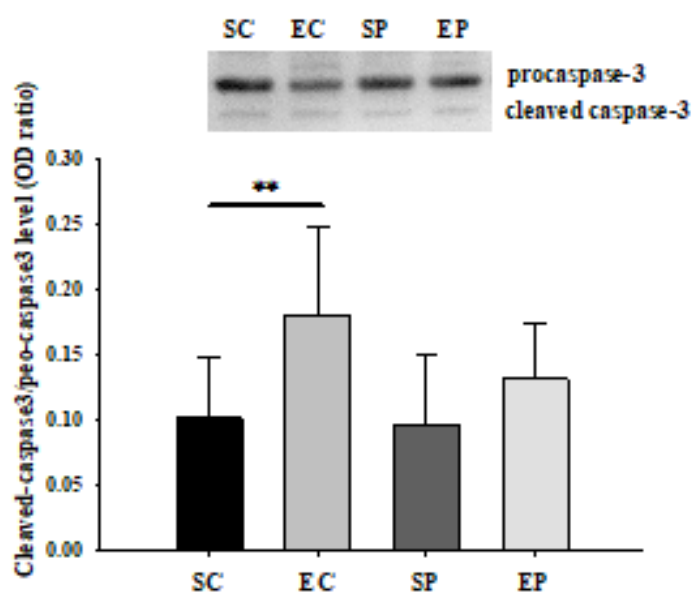

(B)

Soleus cleared-caspase9/pro-casp ase9 ler el

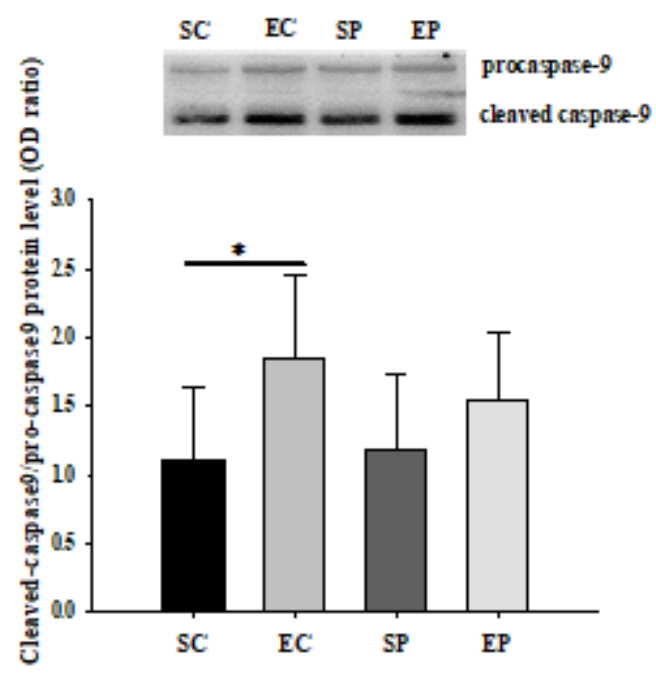

\section{Figure 6}

Effects of excessive exercise and probiotics supplementation on protein contents of Bax/Bcl-2(A), cleaved caspase-9/procaspase-9 (B) and cleaved caspase-3/procaspase-3 (C) in soleus. ${ }^{\star} \mathrm{p} \leq 0.05,{ }^{\star \star} \mathrm{p} \leq$ 0.01 . 